\title{
UNA NUEVA ESPECIE DE BLECHNUM L. (BLECHNACEAE) EN EL NEOTRÓPICO
}

\author{
Alexánder Fco. Rojas Alvarado \\ Jardín Botánico Lankester, Universidad de Costa Rica, apdo. 1031-7050 Cartago, Costa Rica. \\ afrojasa@hotmail.com
}

\begin{abstract}
A new species, Blechnum fuscosquamosum A.Rojas (Blechnaceae) from the Neotropics is here described. It is distinguished from $B$. fragile (Liebm.) C.V.Morton \& Lellinger by its shorter (5-10 mm vs. 8-15 mm), brown to dark brown (vs. brown-yellowish) rhizome scales with acute apex (vs. long attenuate apex), lanceolate to linear-lanceolate (vs. long attenuate) blade, 25-35 pinna pairs [vs. (35-) 40-80 pairs], relatively longer $(4-6.5 \mathrm{~cm}$ vs. $2.8-5.0 \mathrm{~cm})$ pinnae, $1.5-3.0 \mathrm{~mm}$ wide [(vs. $1.0-1.5(-2.0) \mathrm{mm}$ ] fertile pinnae and distribution at higher elevations (2400-3000 $\mathrm{m}$ vs. $800-2100 \mathrm{~m})$.
\end{abstract}

Resumen. Se describe la nueva especie B. fuscosquamosum A.Rojas (Blechnaceae) de la región neotropical. La especie más cercana es $B$. fragile (Liebm.) C.V.Morton \& Lellinger, con la cual se compara.

Palabras Clave / Key Words: Pteridophyta, Blechnaceae, Blechnum, región neotropical.

El género Blechnum L. es cosmopolita y tiene $c a$. 150 especies, según Moran (1995a). El mismo autor cita 22 especies y dos híbridos en Costa Rica. Rojas (1996) describe una especie nueva y menciona la existencia de un híbrido más; más tarde registra otra especie en Costa Rica (Rojas 2001), mientras que Lellinger (2003) segrega una subespecie de $B$. lherminieri (Bory) C.Chr. y Mickel \& Smith (2004) pasan a categoría de especie la subespecie anterior. Se consultaron distintos tratamientos taxonómicos de Blechnaceae en Mesoamérica, Sudamérica y el Caribe (Proctor 1985, 1989, Mickel \& Beitel 1988, Mickel \& Smith 2004, Moran 1995a, 1995b, Moran \& Øllgaard 1995, Rojas 1996, 2001, Smith 1995, Tryon \& Stolze 1993, Lellinger 2003) y se revisaron las colecciones del Herbario Nacional de Costa Rica (CR), del Instituto Nacional de Biodiversidad (INB), de Missouri Botanical Garden (MO) y de University of California (UC). Uno de los resultados es la especie nueva que se describe a continuación.

Blechnum fuscosquamosum A. Rojas, sp. nova Fig. 1

Tipo: Costa Rica. San José: Dota, Cordillera de Talamanca, camino a Providencia de Dota, entrando por Ojo de Agua, frente al restaurante Chespiritos, 9³7'00”'N, 834910”'W, 2500-2950 m, 16 jul 1996, A. Rojas \& M. Coto 2732 (Holotipo: CR, isotipo: INB).

A Blechno fragili rhizomatis squamis brevioribus, pardalibus, apice acuto, pinnis sterilibus longioribus, distributione altitudinali divergens.

Hemiepifita; rizoma de 5-10 $\mathrm{mm}$ de diámetro, largamente rastrero; escamas del rizoma de 5-10 x 0.7-1.0 $\mathrm{mm}$, linear-lanceoladas, bicoloras, el centro pardo oscuro a negruzco y el margen pardo a pardo oscuro, margen denticulado, ápice agudo; hojas estériles y fértiles dimorfas; peciolo de 10-23 cm de largo, pajizo adaxialmente, pajizo a pardo oscuro abaxialmente; lámina 25-65 x 3-10 (-14) cm, pinnatisecta a pinnada, glabra en ambas superficies, gradualmente reducida hacia la base, a veces con lóbulos separados; segmentos 25-35 (-50) pares, 4-6.5 x 1-1.7 cm, adnatos, no involutos, enteros; pardo-amarillentos, glabros; costas pajizas, glabras; aeróforos ausentes; hojas fértiles 3075 x 8-12 cm; pecíolo $10-40 \mathrm{~cm}$, pardo-amarillento a pardo oscuro; pinnas 5-7 x 0.15-0.30 cm.

Distribución: Cordillera de Talamanca y Cordillera de los Andes, 2200-3200 m.

Paratipos: Costa Rica. Limón: Cordillera de Talamanca, Atlantic slope, unnamed cordillera between the Río Terbi and the Río Siní, 900-12’N, 82 $58-59^{\circ} \mathrm{W}$, 2400-2750 m, 13 Sept 1984, G. Davidse et al. 29050 (CR, MO). Puntarenas: Coto Brus, Cerros Tararia, Valle del Silencio, sector de acampar a los jardines, 0907'15”N, 8257'55”'W, 2500 m, 14 abr 1996, F. Quesada 1417 (INB, MO). San José: Pérez Zeledón, Parque Nacional Chirripó, Cordillera de Talamanca, entre Llano Bonito y antes de Monte Sin Fe, $9^{\circ} 26^{\prime} 55^{\prime}$ 'N, 
83³2’05”'W, 2600-2900 m, 29 jul 1996, A. Rojas 2885 (CR, INB, MO).

Otros Especímenes Estudiados: Costa Rica. Cartago: Paraíso, investigation area of the CATIE, $9^{\circ} 33^{\prime} 30^{\prime} \mathrm{N}$, 8341'30”'W, 2600-2700 m, 20 Dic 1990, J. Bittner 159 (CR); El Guarco, Reserva Forestal Río Macho, Cuenca del Reventazón, Estación Ojo de Agua, Sendero La Quebrada, 9³7'55”N, 8349'49”W, 2800-2900 m, 16 ene 1997, B. Gamboa 976 (CR, INB); Cerros Cuericí, 2940 m, 9 nov 1988, Y. Widmer 398 (CR). Limón: Cordillera de Talamanca, atlantic slope, Valle del Silencio, area just $\mathrm{N}$ of Cerro Hoffmann, $4 \frac{1}{2}$ airline $\mathrm{km} \mathrm{W}$ of the Costa Rican-Panamanian border, $9^{\circ} 08^{\prime} \mathrm{N}$, $82^{\circ} 58^{\prime} \mathrm{W}, 2350-2450 \mathrm{~m}, 8$ Sept 1984, G. Davidse et al. 28676 (CR, MO). Limón/Puntarenas: Sendero a Cerro Kámuk, entre Cerro Kasir y Laguna Seca, $9^{\circ} 10^{\prime} \mathrm{N}$, 830'' 2600 m, 11 nov 1996, A. Rojas 3263 (INB).

Puntarenas: Cordillera de Talamanca, upper slopes of Cerro Echandi, ca. $9^{\circ} 01^{\prime} 30^{\prime \prime} \mathrm{N}, 82^{\circ} 49^{\prime} \mathrm{W}, 2700-3000 \mathrm{~m}$, 23 Oct 1983, G. Davidse et al. 23997 (CR, MO); Coto Brus, Sabalito, Fila Cedro, Sendero a Cerro Echandi, a orilla de Quebrada Buru, 901 'N, 8249’ W, 2700-3000 m, 12 oct 1997, B. Gamboa 1712 (INB); Coto Brus, Cerros Tararia, Valle del Silencio, Cerro Asidbeta, 0907'15”N, 82 $57^{\prime} 55^{\prime \prime} \mathrm{W}, 2698 \mathrm{~m}, 6$ jun 1996, M. Moraga 382 (INB); Coto Brus, Sabalito, Las Alturas de Cotón, Estación Biológica Las Alturas, sendero a Cerro Echandi, postes $63-83,9^{\circ} 00^{\prime} \mathrm{N}, 82^{\circ} 49^{\prime} \mathrm{W}, 2400$ 3160 m, 8 ene 1994, A. Rojas 934 (INB).

PANAMÁ. Bocas del Toro: Upper Río Colubre, 25003000 m, Aug 1983, L. Gómez et al. 21927 (CR).

COLOMBIA. Antioquia: Jardín, Alto de Ventanas, $15 \mathrm{~km}$ SO de Jardín en la vía a Riosucio, $5^{\circ} 30^{\prime} \mathrm{N}$, $75^{\circ} 40^{\prime} \mathrm{W}$, R. Callejas et al. 3947 (UC); Jardín, S of Jardín in southern part of department, $2750 \mathrm{~m}, 5^{\circ} 30^{\prime} \mathrm{N}$, $75^{\circ} 50^{\prime} \mathrm{W}, 29$ Oct 1988, G. McPherson et al. 12911 (MO, UC). Bogotá: 2800 m, Lindig 114 (UC).

Cundinamarca: $2400-3000 \mathrm{~m}, 4^{\circ} 35^{\prime} \mathrm{N}, 73^{\circ} 40^{\prime} \mathrm{W}$, ago-dic 1990, A. Repizzo y Z. Calle 145 (MO). Nariño: Pasto, El Encanto, vereda San José Alto, $01^{\circ} 09^{\prime} \mathrm{N}$, $77^{\circ} 10^{\prime} \mathrm{W}, 3400 \mathrm{~m}, 16$ febr 1991, B. Ramírez 3052 (UC).

Putumayo: W side of Laguna de la Cocha, vicinity of Buenavista, 2900-3000 m, 24-26 Oct 1944, J. Ewan 16350, 16352 (UC). Santander: Cordillera Oriental, 5 $\mathrm{km}$ NE of Coromoro or $17 \mathrm{~km}$ ENE of Charala, 2300 m, 8 May 1944, J. Ewan 15666 (UC).

VENEZUELA. Táchira: Cerro San Isidro, $7^{\circ} 34^{\prime} \mathrm{N}$, $72^{\circ} 25^{\prime} \mathrm{W}, 2200-2450 \mathrm{~m}, 13$ Nov 1982, G. Davidse \& A. González 22068 (MO). Trujillo: road to Guaramacal from Bocono, $\mathrm{E}$ side of the mountain, roadside, 9000 ft. [2745 m], 26 Dec 1986, A. Fay 1608 (UC).
ECUADOR. Azuay: The Eastern Cordillera, 1-8 km N of the Vellage of Sevilla de Oro, 8000-9000 ft. [24402745 m], 27 July-12 Aug 1945, W. Camp 4380 (UC). Cañar: 2830 m, 2 Mar 1945, Fosberg \& Prieto 22728 (US). Carchi: Carchi, Tulcán, trail Olivos to Moran, 3250 m, 12 July 1935, Y. Mexía 7484 (US, UC); Uribante, entre El Hato y Portachuelo, ca. $8^{\circ} 05-10^{\prime} \mathrm{N}$, $71^{\circ} 53^{\prime} \mathrm{W}, 2300-2600 \mathrm{~m}, 22$ nov 1985, F. Ortega \& H. Werff 2881 (UC); Espejo, $0^{\circ} 51^{\prime} \mathrm{N}, 7^{\circ} 07^{\prime} \mathrm{W}, 2650 \mathrm{~m}$, 21 ago 1994, $W$. Palacios 12601 (MO, QCNE); Tulcan, $0^{\circ} 35^{\prime} \mathrm{N}, 77^{\circ} 42^{\prime} \mathrm{W}, 3000 \mathrm{~m}, 8$ jul 1992, G. Tipaz et al. 1577 (MO); along road Julio Andrade-El CarmeloTulcan, $0^{\circ} 40^{\prime} \mathrm{N}, 7^{\circ} 38^{\prime} \mathrm{W}, 2900-3200 \mathrm{~m}, 7$ Aug 1989, H. Werff \& E. Gudiño 10977 (MO, UC). Imbabura: Cotacachi, $0^{\circ} 20^{\prime} \mathrm{N}, \quad 78^{\circ} 26^{\prime} \mathrm{W}, 2500-3000 \mathrm{~m}, 26$ nov 1992, E. Gudiño \& B. Cuamacás 1992 (MO); Cotacachi, Parroquia Plaza Gutiérrez, Tabla Chupa, arriba de Apuela, $0^{\circ} 20^{\prime} \mathrm{N}, 78^{\circ} 30^{\prime} \mathrm{W}, 2300-3000 \mathrm{~m}$, 12 mayo 1992, G. Tipaz et al. 1015 (MO, QCNE); Imbabura, 2865 m, 21 Jul 1944, Wiggins 10436 (US). Loja: Loja, 2900 m, 20 Sept-16 Nov 1989, Bogh 47846 (AAU); Loja, 2800-3000 m, 16-19 Nov 1989, Bogh 86628 (AAU, MO); Loja, 2900 m, 1 Febr-20 Apr 1989, B. Eriksen 91202 (AAU); 2500-2910 m, J. Jaramillo et al. 3769 (MO); km 51 on Pan American Hwy. N of Loja, $03^{\circ} 45^{\prime}$ S, $79^{\circ} 15^{\prime}$ W, 2900 m, 1 May 1973, L. Holm-Nielsen et al. 4654 (UC); 2850-2950

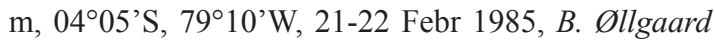
et al. 57826 (AAU); Parque Nacional Podocarpus, the pass road Yangana Valladolid (Nudo de Sabanilla), 042ㄱ'S, 7908'W, 2750-2900 m, 28 Febr 1985, B. Øllgaard et al. $58429(\mathrm{MO}) ; 04^{\circ} 05^{\circ} \mathrm{S}, 79^{\circ} 10^{\prime} \mathrm{W}, 2800$ 3000 m, 13-14 May 1988, B. Øllgaard et al. 74123 (AAU); Loja, Parque Nacional Podocarpus, bosques de Ceja de Montaña en Cerro Toledo, carretera YanganaCerro Toledo, $04^{\circ} 23^{\prime} \mathrm{S}, 7^{\circ} 08^{\prime} \mathrm{W}, 2900 \mathrm{~m}$, ene 1995 , W. Palacios \& M. Tirado 12909 (MO, QCNE, UC).

Napo: Along road between Baeza and Tena, $28 \mathrm{~km} \mathrm{~S}$

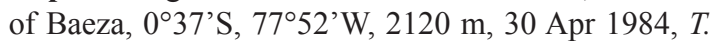
Croat 58751 (MO); carretera Papallacta-Baeza, entre Cuyaya y Papallacta, $00^{\circ} 23^{\prime} \mathrm{S}, 78^{\circ} 01^{\prime} \mathrm{W}, 2750 \mathrm{~m}, 7$ jun 1988, W. Palacios 2580 (MO, UC); Quijos, Reserva Ecológica Antisana, Cordillera de Los Guacamayos, cruce del oleoducto de la compañía ARCO, $0^{\circ} 38^{\prime} \mathrm{S}$, $77^{\circ} 51^{\prime} \mathrm{W}, 2400 \mathrm{~m}, 6-10$ ene 1999, H. Vargas \& E. Narváez 3391 (MO, QCNE, UC); Quijos, Valle Alto del Río Quijos, $5 \mathrm{~km}$ al Sur de Cuyuja, Finca Agroecológica Antisana, $00^{\circ} 28^{\prime} \mathrm{S}, 78^{\circ} 03^{\prime} \mathrm{W}, 2850 \mathrm{~m}$, 16 jun 1998, H. Vargas et al. 1889 (MO, QCNE, UC). Pichincha: Pichincha, $2400 \mathrm{~m}$, Mille 156 (US). Zamora-Chinchipe: $04^{\circ} 27^{\prime} \mathrm{S}, 7^{\circ} 08^{\prime} \mathrm{W}, 2700 \mathrm{~m}$, 14 Febr 1989, B. Øllgaard \& Madsen 90580 (AAU, 
QCA); Zamora-Chinchipe, 2100-2400 m,15 Oct 1943, J. Steyermark 54685 (US).

PERÚ. Amazonas: Chachapoyas, Leymebamba, alre-dedor de la Laguna de Los Cóndores, $6^{\circ} 51^{\prime} \mathrm{S}$, $77^{\circ} 40^{\prime} \mathrm{W}, 2500-2700 \mathrm{~m}, 16$ ago 1998, V. Quipuscoa et al. 1265 (UC); Bongara, outside Laguna de

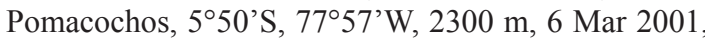
H. Werff et al. 16823 (MO, UC). Cajamarca: Jaén, Sallique, La Cocha, 540'58”S, 79¹4'53”W, 2960 m, 21 jun 1998, J. Campos et al. 5034 (MO, UC); San Ignacio, Tabaconas, El Pajonal, camino al Páramo y al Cerro Coyona, 517'30'S, 79¹6'02'W, $2250 \mathrm{~m}$, 18 nov 1998, J. Campos et al. 5722 (MO); Cutervo, San Andrés de Cutervo, Parque Nacional de Cutervo, caserío "Pajonal", hacia la fila, camino hacia Jaén (Chorro Blanco), 10 ago 1987, C. Díaz y H. Osores 2596 (MO); Urubamba, Machu Pichu, 1309's, 72³1'W, 2900 m, 26 oct 1987, P. Núñez 8392 (MO).

Puno: Carabaya, Ollachea, above falls, 14 Aug 1980, J. Boeke \& S. Boeke (MO).

BOLIVIA. Cochabamba: José Carrasco Torrico, 4 $\mathrm{km}$ desde Liberia hacia Karahuasi, $17^{\circ} 48^{\prime} \mathrm{S}, 64^{\circ} 41^{\prime} \mathrm{W}$, 2350 m, 14 oct 1996, M. Kessler et al. 8993 (UC). La Paz: Nor Yungas, Estación Biológica de Tunquini, senda nueva del camino de la mina (curva al lado O) al pantanón, $16^{\circ} 11^{\prime} \mathrm{S}, 67^{\circ} 53^{\prime} \mathrm{W}, 3000 \mathrm{~m}, 14 \mathrm{Sept}$ 2000, K. Bach et al. 1060 (UC); Nor Yungas, along road between Unduavi and Chulumani, ca. $5 \mathrm{~km}$ beyond Aceromarca, $16^{\circ} 20^{\prime} \mathrm{S}, 67^{\circ} 38^{\prime} \mathrm{W}, \mathrm{ca} .2800 \mathrm{~m}$, 25 Nov 1980, T. Croat 51475 (MO, UC); Sud Yungas, La Paz-Chulumani road, $15.1 \mathrm{~km}$ W of Chulumani, $9.3 \mathrm{~km}$ from Huancané, $16^{\circ} 15^{\prime} \mathrm{S}, 67^{\circ} 30^{\prime} \mathrm{W}, 2450 \mathrm{~m}$, 2 Aug 1989, A. Fay \& L. Fay 2501 (MO, UC); Nor Yungas, Trocha al Valle de Coscapa, Parque Nacional Cotapata, $16^{\circ} 12^{\prime} \mathrm{S}, 67^{\circ} 53^{\prime} \mathrm{W}, 3250 \mathrm{~m}, 11$ sept $1997, M$. Kessler et al. 11824 (UC); Yungas, $16^{\circ} 08^{\prime} \mathrm{S}, 68^{\circ} 07^{\prime} \mathrm{W}$, 2900 m, 18 Mar 1987, J. Solomon 16392 (MO). Santa Cruz: Manuel M. Caballero, Siberia, 17050'12” S, $64^{\circ} 42^{\prime} 05^{\prime \prime} \mathrm{W}, 2450 \mathrm{~m}, 24$ mar 2004, $R$. Núñez 562

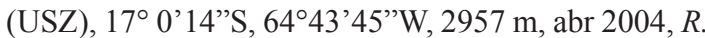
Núñez 622 (USZ).

La especie difiere de Blechnum fragile por las escamas del rizoma más cortas $(5-10 \mathrm{~mm}$ de largo vs. 8-15 $\mathrm{mm}$ ), pardas a pardo oscuro (vs. pardo-amarillentas a pardas), con el ápice agudo (vs. largamente atenuado), lámina lanceolada a linear-lanceolada (vs. linear), 2535 pares de pinnas (hasta 50 pares en Sudamérica) [vs. (35-) 40-80 pares], pinnas estériles relativamente más largas (4-6.5 cm de largo vs. $2.8-5.0 \mathrm{~cm}$ ), pinnas fértiles de 1.5-3.0 mm de ancho (vs. 1.0-1.5 (-2.0) $\mathrm{mm}$ ] y por hallarse a mayor elevación (2200-3200 m vs. 800-2100 m). En la especie nueva no hay mucha diferencia de longitud entre las pinnas fértiles y las estériles, pero en $B$. fragile las fértiles pueden ser hasta dos veces más largas que las estériles (Fig. 1).

Por el tamaño más desarrollado de las pinnas, Blechnum fuscosquamosum se parece al híbrido entre Blechnum ensiforme (Liebm.) C.Chr. y B. fragile (Liebm.) C.V. Morton ex Lellinger (Mickel \& Beitel 1988, Moran 1995), el cual se localiza desde México hasta el norte de Costa Rica, pero la nueva especie difiere por tener escamas del rizoma pardo-oscuras con ápice agudo (vs. amarillentas con ápice linear), base de la lámina gradualmente reducida (vs. abruptamente reducida), pinnas basales cercanas entre sí (vs. distantes) y se localiza a 2200-3200 m (vs. 900-2100 m).

Etimología. El epíteto fuscosquamosum se refiere a las escamas pardas del rizoma.

Agradecimientos. Agradezco a los herbarios CR, INB, MO y UC por permitirme utilizar sus colecciones, a Carlos O. Morales por sus correcciones del latín y a los revisores anónimos por los comentarios sobre el manuscrito.

\section{Literatura Citada}

Lellinger, D.B. 1989. The ferns and fern-allies from Costa Rica, Panama, and The Chocó. Part I. Pteridologia. p. 254-264.

Lellinger, D.B. 2003. Nomenclatural and Taxonomic Notes on the Pteridophytes of Costa Rica, Panama, and Colombia, III. Amer. Fern J. 93 (3): 146-151.

Mickel, J.T. \& J.M. Beitel. 1988. Pteridophyte Flora of Oaxaca, México. Mem. New York Bot. Gard. 46: 79-89.

Mickel, J.T. \& A.R. Smith. 2004. The Pteridophytes of Mexico. Mem. New York Bot. Gard. 88: 1054 p.

Moran, R.C. 1995a. Blechnaceae. In: Moran, R.C. \& R. Riba (eds.). Flora Mesoamericana. Vol. 1. Psilotaceae a Salviniaceae. Univ. Nac. Autónoma de México. p. 325-333.

Moran, R.C. 1995b. Five new species and two new combinations of ferns (Polypodiopsida) from Ecuador. Nord. J. Bot. 15 (1): 49-58.

Moran, R.C. \& B. Øllgaard. 1995. Six new species of ferns (Polypodiopsida) from Ecuador. Nord. J. Bot. 15 (2): 177-185.

Proctor, G.R. 1985. Ferns of Jamaica, a guide to the Pteridophytes. British Museum. 631 p.

Proctor, G.R. 1989. Ferns of the Puerto Rico and The 
Virgin Islands. Mem. New York Bot. Gard. 53: 169-175.

Rojas, A.F. 1996. Aportes a la Flora Pteridophyta Costarricense. II. Taxones nuevos. Brenesia 45-46: 33-50.

Rojas, A.F. 2001. Seis especies nuevas y dos nuevos registros de helechos (Pteridophyta) para Costa Rica. Rev. Biol. Trop. 49 (2): 435-452.
Smith, A.R. 1995. Blechnaceae. In: Berry, P.E., B.K. Holst \& K. Yatskievych (eds.). Flora of the Venezuelan Guayana. Vol. 2. Pteridophytes, Spermatophytes, Acanthaceae-Araceae. Oregon, Timber Press. p. 23-29.

Tryon, R.M. \& R.G. Stolze. 1993. Pteridophyta of Perú. Part V. 18. Aspleniaceae - 21. Polypodiaceae. Fieldiana, Bot., n.s. 32: 54-70.

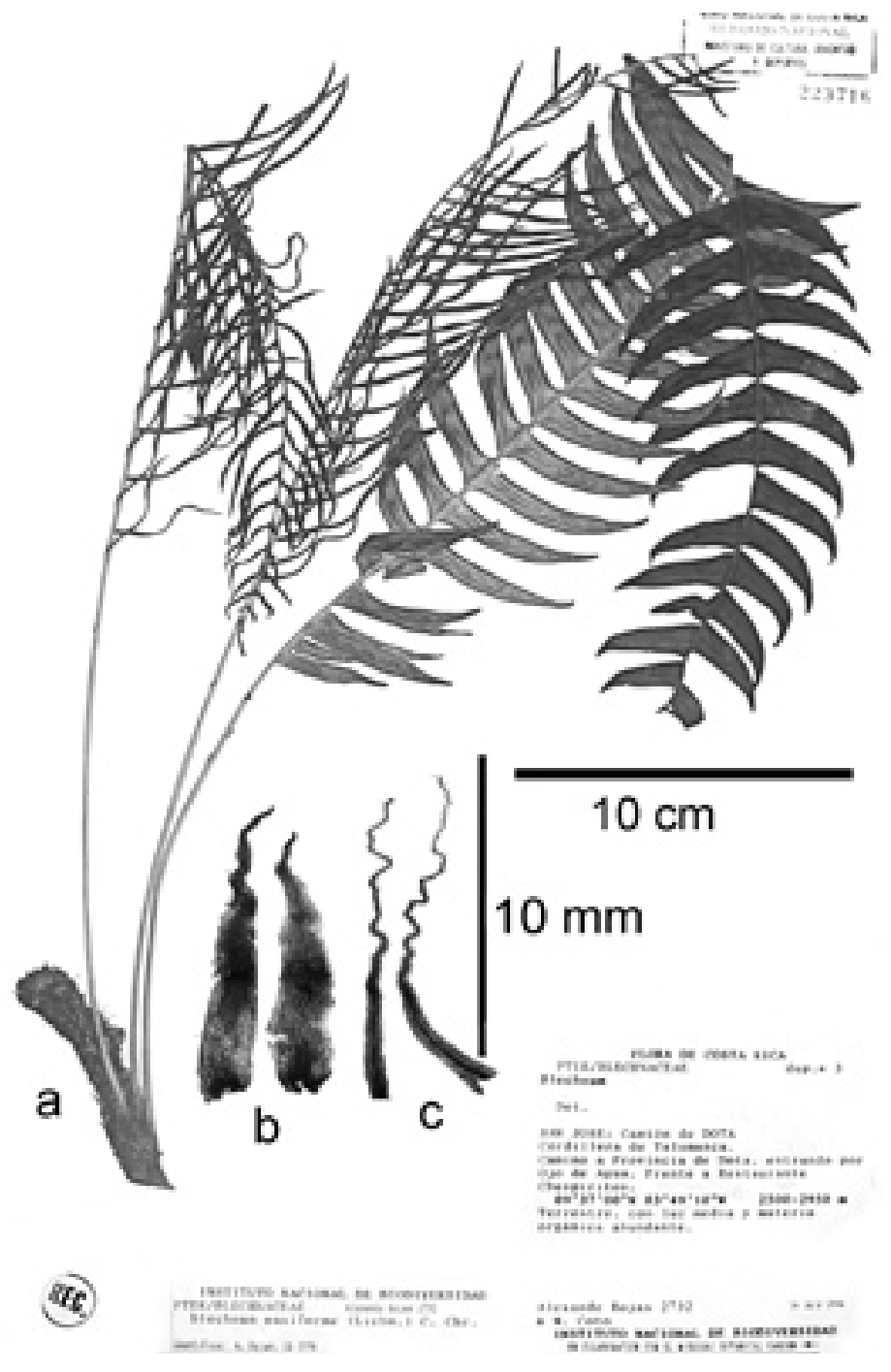

Fig. 1. a-b. Blechnum fuscosquamosum (A. Rojas \& M. Coto 2732, CR). a. Hábito. b. Escamas del rizoma. c. Blechnum fragile, escamas del rizoma (M. Grayum 9289, CR). 